\title{
Características de la personalidad de jóvenes jugadores de baloncesto (15-under)
}

\section{Characteristics in the Personality of Young Basketball Players (15 and under)}

Ana Alicia Marín*, Ma Isabel Piñar*, Pablo Camacho*, Vicente Brox*, Ma Teresa Miranda-León*, Ernesto Suárez-Cadenas*, Francisco Alarcón** y David Cárdenas*

*Universidad de Granada **Universidad Católica San Antonio de Murcia

Resumen: El objetivo de este trabajo fue estudiar los rasgos de personalidad de los jóvenes jugadores de baloncesto de 13-15 años $(\mathrm{n}=54)$ a través de la evaluación de las dimensiones del cuestionario de personalidad BFQ-NA, para niños y adolescentes. Se trata de un estudio no experimental en el que se emplea un diseńo transeccional o transversal descriptivo. Los resultados obtenidos permiten afirmar que los jugadores seleccionados para la muestra se caracterizan por ser personas: a) perseverantes, tenaces y exigentes, b) abiertas a la novedad, creativas, espontáneas y cultas, c) con valores normales de sociabilidad, seguridad y entusiasmo, d) amables, honestas, educadas y e) tranquilas, serenas y poco irritables.

Palabras clave: Personalidad, Big-five model, jóvenes, baloncesto.
Abstract: The aim of the present work was to assess the personality traits of young basketball players aged $13-15$ years $(n=54)$ through the evaluation of the dimensions of personality questionnaire BFQ-NA, for children and adolescents. We used a descriptive transversal design in this study which was non-experimental. The results that were obtained confirm that the players selected for the sample are characterized by being: a) persistent, tenacious and demanding, b) open to novelty, creative, spontaneous and educate, c)with normal values of sociability, confidence and enthusiasm, d) friendly, honest and polite, e) calm, serene and slightly irritable.

Key words: Personality, Big-Five model, young players, basketball.

\section{Introducción}

Las relaciones entre personalidad y deporte han sido objeto de estudio a lo largo de las últimas décadas (Bilalic, McLeold y Gobet, 2007; Hoyt, Rhodes, Hausenblass, Giacobbi, 2009; Katjna, Tusak, Baric, Burnick, 2004; Piedmon, Hill y Blanco, 1999; Ruiz, 2005, 2007, 2008; Trinic, Barancic y Nazor, 2008). Según Dosil (2004), "La personalidad es uno de los temas con mayor investigación en psicología, convirtiéndose en uno de los tópicos más importantes en Psicología de la Actividad Física y el Deporte en la década de los 60 y 70" (p.199).

El estudio de la personalidad en el ámbito deportivo y en el extradeportivo como en psicología de las organizaciones (Barrit y Mount, 1991; Hartmann y Grønnerød, 2009; Tett, Jackson y Roshtein, 1991), en psicología de la educación (Graciano y Ward, 1992; Parker y Stumpf, 1998) y en psicología clínica (Costa y McRae, 1991), confluye en el famoso modelo de los "Cinco Grandes Factores de la Personalidad" (MPBFQ). Los test empleados para medir estos Cinco Grandes Factores de la Personalidad han sido, principalmente, el modelo NEO - Personality Inventory (NEO-PI) creado por los autores Costa y McRae (1992), y el cuestionario de personalidad Big Five (BFQ) de Caprara, Barbaranelli, borgogni y Perugini (1993), cuya estructura es apropiada e integradora para describir la personalidad en el lenguaje "natural" y en el contexto de los cuestionarios de personalidad. El análisis

Correspondencia con: Ma Isabel Piñar López. Facultad de Ciencias de la Actividad Física y el Deporte. Carretera de Alfacar s/n, 18071. Granada. E-mail: maribelpinar@ugr.es de la dimensionalidad del BFQ adaptado para niños y adolescentes (BFQ-NA) ha mostrado una estructura de cinco factores que es viable y adecuada de acuerdo con los requisitos psicométricos establecidos cuando los informantes son los propios niños (Soto et al., 2011). Este cuestionario (BFQNA), comprende una fiabilidad cuya taxonomía constituye una herramienta fundamentada y viable para la descripción de la personalidad infantil. Otros autores coinciden con estos datos, poniendo de manifiesto que los cinco factores son una buena representación de la estructura de personalidad infantil y adolescente (Barbaranelli et al., 2003; Goldberg, 2001; Markey et al., 2002).

Tomando como referencia los estudios anteriormente citados, nuestro objetivo es describir los rasgos de la personalidad de los jóvenes jugadores de baloncesto (13-15 años) a través de la evaluación y descripción de las dimensiones del cuestionario de personalidad BFQ-NA (Bermúdez, 1995).

\section{Método}

\section{Participantes}

Los sujetos que formaron parte de este estudio fueron 54 jóvenes jugadores de baloncesto pertenecientes a la cantera del club Cajasol que participó en la liga ACB (Asociación de Clubes de Baloncesto) durante la temporada 2011/12, de entre 13 y 15 ańos de edad $(M=13.43 ; S D=1.5)$. 


\section{Diseño}

Se trata de un estudio no experimental con un diseño transeccional o transversal descriptivo, mediante el cual se analizan las características de personalidad de los jóvenes jugadores de baloncesto (13-15 años).

\section{Material}

El instrumento utilizado es la adaptación al castellano (Bermúdez, 1995) del "Cuestionario de Personalidad BFQ" de Caprara et al. (1993), adaptado para nińos y adolescentes.

El cuestionario BFQ-NA consta de 65 ítems y posee cinco dimensiones graduadas mediante una escala de 5 alternativas: 5 , casi siempre; 4 , muchas veces; 3 , algunas veces; 2 , pocas veces; 1 , casi nunca. Las dimensiones son: Conciencia (Co), que evalúa la autonomía, el orden, la precisión y el cumplimiento de normas y compromisos; Apertura (Ap), que evalúa tanto aspectos intelectuales, esencialmente de tipo escolar, como intereses de tipo cultural, fantasía, creatividad e interés en otras gentes; Extraversión (Ex), que hace referencia a aspectos tales como la creatividad, entusiasmo, autoconfianza o asertividad; Amabilidad (Am), entendida como la preocupación y sensibilidad hacia los otros y sus necesidades, e Inestabilidad emocional (Ie) referida a los sentimientos de ansiedad, depresión, descontento o ira.

\section{Procedimiento}

Se pasó el cuestionario a cada uno de los jugadores una hora antes del entrenamiento. Previamente, se obtuvo el consentimiento informado de los padres. Todos los jugadores recibieron las mismas instrucciones y fueron evaluados en las mismas condiciones. Cada jugador obtuvo una puntuación directa para cada dimensión de la personalidad que, posteriormente convertimos en puntuaciones transformadas para su adecuada interpretación.

\section{Resultados}

En la tabla 1 se presentan los valores descriptivos de las dimensiones analizadas.

Tabla 1. Medidas descriptivas de las dimensiones de BFQ-NA para jóvenes jugadores ( $\mathrm{n}=54)$.

\begin{tabular}{lcccc}
\hline Cuestionario BFQ-NA & Mínimo & Máximo & Media & Desv. típ. \\
\hline Co & 56 & 99 & 77,61 & 8,916 \\
Ap & 22 & 40 & 32,70 & 4,183 \\
Ex & 30 & 50 & 42,54 & 4,940 \\
Am & 30 & 49 & 39,61 & 4,644 \\
Ie & 10 & 43 & 23,70 & 6,675 \\
\hline
\end{tabular}

\section{Discusión y conclusiones}

Los resultados que obtuvimos, después de hacer las correspondientes transformaciones de puntuación a la escala BFQNA, muestran que los jugadores de la muestra seleccionada presentan valores normales de las dimensiones: Extraversión (Ex) y Amabilidad (Am), valores altos y muy altos para las dimensiones Conciencia (Co) y Apertura (Ap) y valores bajos y muy bajos para la dimensión Inestabilidad emocional (Ie). Esto permite afirmar que se trata de personas: a) perseverantes, tenaces y exigentes, b) abiertas a la novedad, creativas, espontáneas y cultas, c) con valores normales de sociabilidad, seguridad y entusiasmo, d) amables, honestas, educadas y e) tranquilas, serenas y poco irritables. No se aprecia demasiadas diferencias entre las categorías, si bien, en la dimensión Apertura (Ap), la categoría Mini obtiene diferencias significativas con la categoría Cadete y en la dimensión Extraversión (Ex), existen diferencias significativas entre la categorías Infantil y Cadete.

\section{Referencias bibliográficas}

Barrick, M. R., y Mount, M. K. (1991). The big five personality dimensions and job performance: A meta-analysis. Personnel Psychology, 44, 1-26.

Bermudez, J. (1995). Cuestionario "Big Five”. Adaptación al castellano del cuestionario BFQ de Caprara, Barbaranelli, \& Borgogni (1993). Madrid: Tea Ediciones.

Bilalic M., McLeold, P. y Gobet, F. (2007). Personality profiles of young chess players. Personality and Individual Differences, 42 (6), 901-910.

Caprara, G. V., Barbaranelli, C., Borgogni, L. y Perugini, M. (1993). The Big Five Questionnaire: A new Questionnaire for the measurement of the five factor model. Personality and Individual Differences, 15, 281288.

Costa, P. T. y McCrae, R. R. (1990). Personality disorders and the fivefactor model of personality. Journal of Personality Disorders.4, 362-371.

Costa, P. T., y McCrae, R. R. (1992). Revised NEO Personality Inventory (NEO-PI-R) and NEO Five-Factor Inventory (NEO-FFI) professional manual. Odessa, FL: Psychological Assessment Resources.
Graciano, W. G. y Ward, D. (1992). Probing the Big Five in Adolescence: Personality and Adjustment during a Developmental Transition. Journal of Personality, 60, 425-439.

Hartmann, E. y Grønnerød, C. (2009). Rorschach variables and Big Five scales as predictors of military training completion: a replication study of the selection of candidates to the naval special forces in Norway. Journal of personality assessment, 91 (3), 254-64.

Hoyt, A. L., Rhodes, R. E., Hausenblas, H. A., y Giacobbi, P. R. (2009). Integrating fivefactor model facet level traits with the theory of planned behavior and exercise. Psychology of Sport and Exercise, 10, 565-572.

McRae, R. R. y Costa, P. T. (1987). Validation of the five factor model of personality across instrument and observers. Journal of Personality, 60, $175-215$.

Parker, W. D. y Stumpf, H. (1998). A validation of the five-factor model of personality in academically talented youth across observers and instruments. Personality and Individual Differences 25 (6), 1005-1025. 
Piedmon, R. L., Hill, D. C., y Blanco, S. (1999). Predicting athletic performance using the five factor model of personality. Personality and Individual Differences, 27, 769-777.

Piñar, M.I., Cárdenas, D., Alarcón, F., Escobar, R., Estevez, F. y Torre, E. (2009). Characteristics of the dimensions and sub-dimensions of young basketball players' personalities. Revista de Psicología del Deporte, 18, 415-419.

Ruiz, R. (2005). Análisis de diferencias de personalidad en el deporte del judo a nivel competitivo en función de la variable sexo y categoría de edad deportiva. Cuadernos de Psicología del Deporte,5 (1), 29-48.

Ruiz, R. (2008). Aportaciones del análisis subdimensional del cuestionario de personalidad BFQ para la predicción del rendimiento en judokas jóvenes de competición. Cuadernos de Psicología del Deporte, 8 (1), 5-29. Soto, G., Ferrándiz, C., Sáinz, M., Ferrando, M., Prieto, M.D., Bermejo, R. y Hernández, D. (2011). Características psicométricas del cuestionario de personalidad BFQ-NA (Big Five Questionnaire - Nińos y Adolescentes). Aula Abierta, 39 (1), 13-24.

Stoll, O. Lau, A. y Stoeber, J. (2008). Perfectionism and performance in a new basketball training task: Does striving for perfection enhance or undermine performance? Psychology of sport and Exercise, 9, 620-629.

Trinić, V. Barančić, M. y Nazor, M. (2008). The five factor model of personality and aggressiveness in prisoners and athletes. Kinesiology, 40 (2), 170-181. 